Original paper

\title{
Diagnostic yield of magnetic resonance imaging for cholangiocarcinoma in primary sclerosing cholangitis: a meta-analysis
}

\author{
Jinendra Satiya' ${ }^{1}$ Omar Y Mousa², Kapil Gupta' ${ }^{1}$, Shivani Trivedi', Sven P Oman³ , Karn Wijarnpreecha', \\ Denise M Harnois ${ }^{3}$, Juan Enrique Corral ${ }^{3}$ \\ 'Department of Internal Medicine, University of Miami/JFK Medical Center, West Palm Beach, FL, Unites States \\ 2Department of Hepatology, Mayo Clinic, Rochester, MN, Unites States \\ ${ }^{3}$ Department of Gastroenterology, Mayo Clinic, Jacksonville, FL, Unites States
}

\begin{abstract}
Aim of the study: Combined magnetic resonance imaging and magnetic resonance cholangiopancreatography (MRI/MRCP) can identify biliary strictures and diagnose primary sclerosing cholangitis (PSC). Diagnosis of cholangiocarcinoma in patients with PSC remains challenging, and the accuracy of MRI/MRCP has not been completely established. We aimed to determine the sensitivity and specificity of MRI/MRCP in the diagnosis of cholangiocarcinoma among patients with PSC from the published literature.

Material and methods: We searched Embase, PubMed, Cochrane, Scopus, ClinicalTrials.gov, and abstracts from relevant scientific meetings and performed a systematic review and meta-analysis to estimate the diagnostic yield of MRI/MRCP in patients with PSC. Sensitivity and specificity were calculated from pooled estimates of cholangiocarcinoma cases identified and lesions missed. Modifying variables were included in a meta-regression model.

Results: Our literature search yielded 302 articles and 9 conference abstracts; 8 studies involving 846 liver patients from 5 countries were included in the final analysis. Of those, 531 had PSC and received MRI/MRCP. Thirty-six (6.8\%) patients were diagnosed with cholangiocarcinoma ( 33 true positive, 3 false negative and 1 false positive). Pooled sensitivity was $98.9 \%$ (95\% Cl: 98.6-99.3\%). Cholangiocarcinoma cases missed by MRI/MRCP were diagnosed as beading irregularities of the central hepatic ducts, or PSC-related diffuse stricture. Metaregression revealed that neither publication year, study design, nor sample size had a significant effect on observed cancer rates $(p=0.9,0.3$, and 0.3 , respectively).

Conclusions: MRI/MRCP followed by endoscopic retrograde cholangiopancreatography (ERCP) is a sensitive and specific tool to diagnose cholangiocarcinoma among patients with PSC. Further research should estimate MRI/ MRCP diagnostic accuracy for cholangiocarcinoma using prospective methodology and longer term outcomes.
\end{abstract}

Key words: abdominal imaging, cholangiocarcinoma, primary sclerosing cholangitis, screening.

\section{Address for correspondence}

Dr. Juan Enrique Corral, Department of Gastroenterology, Mayo Clinic, 4500 San Pablo Road, Davis Building 6th floor 32224, Jacksonville, United States, e-mail: corral.juan@mayo.edu

\section{Introduction}

Mortality associated with primary sclerosing cholangitis (PSC) is substantial. Patients with PSC have 400 times higher risk of developing cholangiocarcinoma than those without PSC, with a 10-year cumulative cancer risk of $7 \%$ to $9 \%[1,2]$. The incidence rate of intrahepatic cholangiocarcinoma has been increasing in the past 2 decades in North America, Asia, and Europe, with a significant percentage of cases being related to PSC $[3,4]$. The risk of developing cholangiocarcinoma does not appear to be related to the duration of PSC $[2,5]$. In up to half of patients, cholangiocarcinoma is detected simultaneously with PSC 
and occasionally helps establish the initial diagnosis of PSC [2].

Magnetic resonance imaging (MRI) and magnetic resonance cholangiopancreatography (MRCP) identify biliary strictures clearly and, in many cases, reveal cholangiocarcinoma. Although there is agreement that all patients with cirrhosis require abdominal MRIs to screen for hepatocellular carcinoma every 6 months, there is limited evidence of whether a similar approach can be used to identify early cholangiocarcinoma in noncirrhotic stage PSC [6].

American Association for the Study of Liver Diseases (AASLD) guidelines on cancer surveillance in patients with PSC are unclear, and the current recommendation includes "a combination of an imaging study and [cancer antigen] CA 19-9 annually" [6]. All imaging findings suspicious for cholangiocarcinoma (i.e., a malignant-appearing mass with delayed venous phase enhancement) require a positive biopsy or cytology confirmation. Other authors are more specific, recommending combined MRI and MRCP (MRI/MRCP) and serum CA 19-9 levels in patients every 6 or 12 months to screen for cholangiocarcinoma, hepatocellular carcinoma, pancreatic cancer, and gallbladder cancer [7]. CA 19-9 testing has notable shortcomings as a screening or diagnostic tool for cholangiocarcinoma as it tends to increase with many other benign biliary conditions. The sensitivity of CA $19-9$ value greater than $100 \mathrm{U} / \mathrm{ml}$ was only $53 \%$ [8]. Despite these recommendations, the accuracy of $\mathrm{MRI} / \mathrm{MRCP}$ in detecting cholangiocarcinoma in the setting of PSC has not been clearly determined [9]. A previous study suggested that MRI/MRCP has the highest sensitivity (89\%) and specificity (75\%) of all imaging modalities [10].

Our study aimed to estimate the diagnostic yield of MRI/MRCP for cholangiocarcinoma in patients with PSC, with and without cirrhosis, based on the current literature.

\section{Material and methods}

Review methodology and reporting were performed according to the Preferred Reporting Items for Systematic Reviews and Meta-Analyses (PRISMA) guidelines [11]. The literature databases searched included Embase, PubMed (U.S. National Library of Medicine), Cochrane (The Cochrane Collaboration), Scopus (Elsevier), and ClinicalTrials.gov (US National Institutes of Health); we also searched for abstracts from Digestive Disease Week, AASLD, American College of Gastroenterology, and Radiological Society of North America annual meetings. References from
2 relevant meta-analyses were also included for review $[12,13]$. All studies reporting MRI/MRCP performed in patients with PSC were included. No distinction was made between patients with PSC with or without cirrhosis as this information was unavailable in the majority of studies.

The search was performed in PubMed using the following sequence: (primary sclerosing cholangitis [MeSH] AND cholangiocarcinoma [MeSH]) AND magnetic resonance imaging $[\mathrm{MeSH}]$. Studies were limited to human studies published up to November 30, 2018. No language restrictions were used. Analogous strategies were used to search the other 4 databases and the meeting abstracts.

\section{Information coding}

Three investigators (J.S., K.G., S.T.) reviewed manuscripts and abstracts for selection of potentially relevant articles independently. For journal titles, full text articles were retrieved for further review. Titles that could not be associated with an abstract were excluded from the review. Studies with less than 20 patients enrolled, systematic reviews, and economic models were excluded, a priori. Manuscripts that included MRI/MRCP imaging performance in all liver diseases (with PSC as a subgroup) were included in the study. Studies that used biomarkers or imaging tests different from MRI/MRCP (e.g., abdominal ultrasound or computed tomography) as the main diagnostic tool were excluded. The majority of studies required endoscopic biopsies or brushings after positive cross-sectional imaging. If multiple manuscripts studied the same patient population, only the most recent one was included. If they provided complementary information (e.g., different years), all were included. For studies that included patients with different cholestatic disorders, the subgroup of patients with PSC was extracted and analyzed individually. If there was any discrepancy of whether a study should be included, a fourth investigator (J.E.C.) would decide on adequacy.

The following variables were recorded from each article: publication year, author names, country, participant demographics (i.e., sex, age, ethnicity), clinical characteristics (i.e., primary liver disease and indication for imaging), study design (i.e., case series, case control, retrospective cohort, prospective), and final diagnosis (i.e., cholangiocarcinoma vs. benign stricture or benign mass) (Table 1) [14-21]. Authors were not contacted to provide additional information.

Study quality or risk of bias was assessed with the Newcastle-Ottawa Scale scored from 0 to a maximum of 9 points [22]. 
Table 1. Characteristics of studies reporting MRI/MRCP in primary sclerosing cholangitis

\begin{tabular}{|c|c|c|c|c|c|c|c|c|}
\hline First author & $\begin{array}{c}\text { Publication } \\
\text { year }\end{array}$ & Location & Design & Source & Specific population & $\begin{array}{l}\text { Patients } \\
\text { invited }^{a}\end{array}$ & $\begin{array}{l}\text { PSC } \\
\text { Patients } \\
\text { enrolled }\end{array}$ & $\begin{array}{l}\text { PSC } \\
\text { with } \\
\text { MRCP }\end{array}$ \\
\hline Angulo P [14] & 2000 & $\begin{array}{l}\text { United } \\
\text { States }\end{array}$ & Case control & $\begin{array}{l}\text { Embase, } \\
\text { meta-analysis } \\
\text { radiology } 2010\end{array}$ & Suspected PSC & 73 & 23 & 23 \\
\hline Baskan 0 [15] & 2016 & Turkey & Case series & PubMed & $\begin{array}{l}\text { PSC, autoimmune hepatitis, primary biliary } \\
\text { cholangitis, nonalcoholic steatohepatitis, } \\
\text { and portal hypertensive biliopathy }\end{array}$ & 70 & 39 & 39 \\
\hline Berstad AE [16] & 2006 & Norway & Case series & $\begin{array}{l}\text { Embase, } \\
\text { meta-analysis } \\
\text { radiology } 2010\end{array}$ & $\begin{array}{l}\text { PSC, autoimmune hepatitis, primary } \\
\text { biliary cholangitis, hemochromatosis, } \\
\text { nonalcoholic steatohepatitis, and other }\end{array}$ & 66 & 39 & 39 \\
\hline Muir AJ [17] & 2017 & $\begin{array}{l}\text { United } \\
\text { States }\end{array}$ & $\begin{array}{l}\text { Prospective } \\
\text { trial }\end{array}$ & $\begin{array}{l}\text { Embase, } \\
\text { conf. AASLD }\end{array}$ & PSC & 234 & 234 & 234 \\
\hline Samuel MT [18] & 2017 & $\begin{array}{l}\text { United } \\
\text { States }\end{array}$ & $\begin{array}{l}\text { Retrospective } \\
\text { cohort }\end{array}$ & $\begin{array}{l}\text { Embase, } \\
\text { conf. DDW }\end{array}$ & $\begin{array}{l}\text { Cholangiocarcinoma } \\
\text { (with and without PSC) } \\
\text { vs. healthy controls }\end{array}$ & 210 & 20 & 20 \\
\hline Tenca A [19] & 2016 & Finland & Case series & $\begin{array}{l}\text { Embase, } \\
\text { conf. UEGW }\end{array}$ & Intra- and extrahepatic PSC & 50 & 59 & 59 \\
\hline Tenca A [20] & 2018 & Finland & $\begin{array}{l}\text { Retrospective } \\
\text { cohort }\end{array}$ & PubMed & Intra- and extrahepatic PSC & 48 & 48 & 48 \\
\hline Weber C [21] & 2008 & Germany & Case control & PubMed & PSC vs. healthy controls & 95 & 69 & 69 \\
\hline Total & & & & & & 846 & 531 & 531 \\
\hline
\end{tabular}

AASLD - American Association for Study of Liver Disease, conf. - conference, DDW - Digestive Disease Week, MRCP - magnetic resonance cholangiopancreatography, MRI - magnetic resonance imaging, PSC - primary sclerosing cholangitis, UEGW - European Gastroenterology Week

apatients invited include controls and all diseases

\section{Statistical analysis}

The numbers of patients invited to the study, enrolled in the study, and receiving MRI/MRCP were recorded. Only patients receiving MRI/MRCP were analyzed per our protocol.

Detection rates and corresponding 95\% CIs were calculated using the initial sample size and Poisson distribution. We used a random-effects model to pool detection rates. Forest plot graphs were created with 95\% CIs. Two-by-two tables were calculated with true-positive, false-positive, true-negative, and falsenegative estimates. Diagnostic yield of MRI/MRCP was reported as sensitivity, specificity, negative predictive value, and positive predictive value.

Meta-regression evaluated for 3 effect-modifying variables: sample size (arbitrarily > 50 patients), study design (i.e., case series, case control, cohort, prospective) and study population (invited only patients with PSC or different liver conditions). Between-study heterogeneity was quantified using the $I^{2}$ statistic [23]. Publication bias was investigated by visual inspection of funnel plots. All statistical analyses were performed with Stata, version SE 14 (StataCorp LLC).

\section{Results}

Our initial literature search yielded 302 articles and 9 conference abstracts. Forty-two manuscripts and 9 conference abstracts met our inclusion criteria and were retrieved for full review from Embase (81 titles found, 25 retrieved), PubMed (34 found, 12 retrieved), Scopus (20 found, 3 reviewed), Cochrane (5 found, 0 retrieved), ClinicalTrials.gov (4 found, 2 retrieved), as well as Digestive Diseases Week and AASLD proceedings ( 9 found, 9 retrieved). After removing duplicates, 32 studies met inclusion criteria. Twenty-four studies were excluded due to duplicate samples or incomplete data (Fig. 1).

Eight studies involving 846 patients with liver disease from 5 countries were included in the final analysis. Of those, 531 had PSC and all received MRI/ MRCP (Table 1). Three studies were case series, 2 were case control, 2 were retrospective cohorts, and 1 was a prospective trial.

Thirty-four cases (6.4\%) were initially diagnosed with cholangiocarcinoma. Thirty-six cases were confirmed (33 true positive, 1 false positive and 3 false negative [missed by MRI/MRCP]). Pooled sensitivi- 


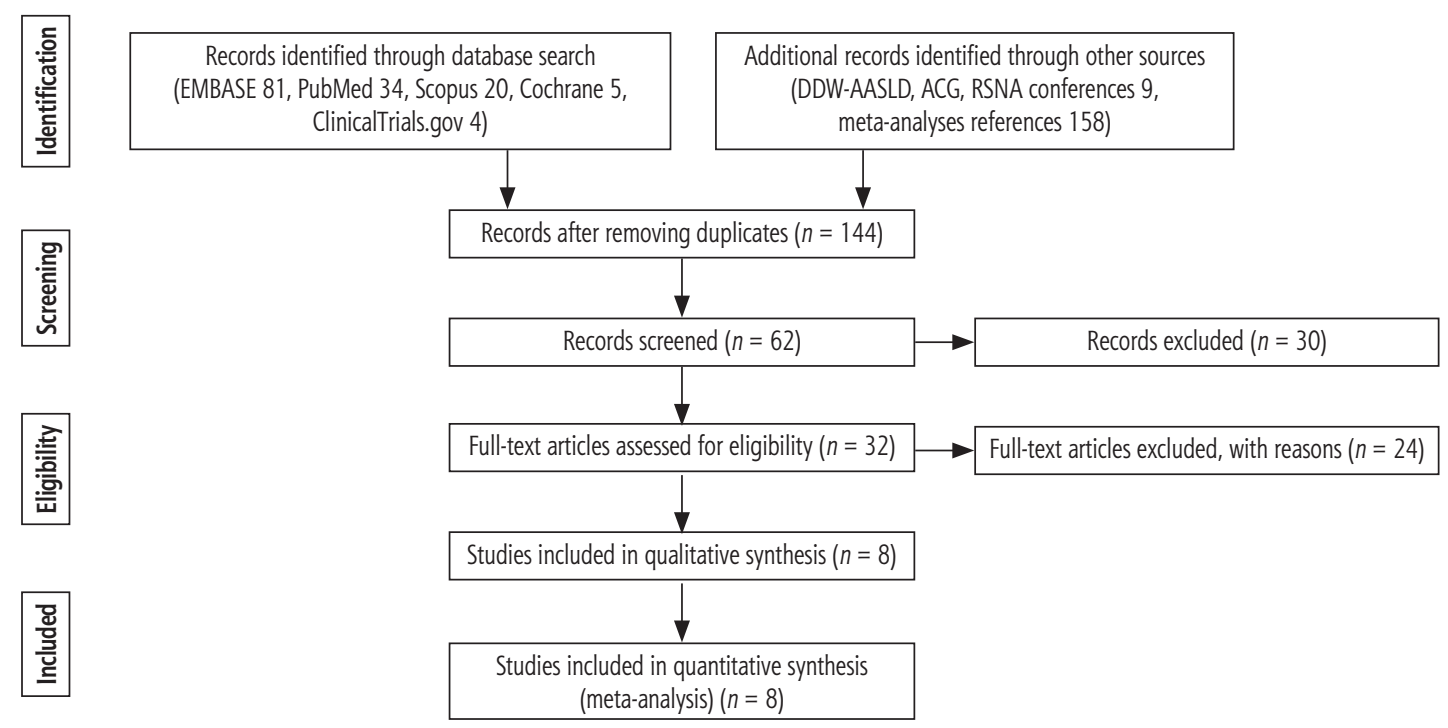

AASLD - American Association for the Study of Liver Diseases, ACG - American College of Gastroenterology, DDW - Digestive Diseases Week, MRCP - magnetic resonance cholangiopancreatography, MRI - magnetic resonance imaging, RSNA - Radiological Society of North America

Fig. 1. Diagnostic yield of MRI/MRCP to identify cholangiocarcinoma in PSC. Study selection flow diagram (PRISMA 2009)

Table 2. Imaging findings and sensitivity estimates

\begin{tabular}{|c|c|c|c|c|c|c|c|c|c|}
\hline First author & $\begin{array}{l}\text { PSC with } \\
\text { MRCP, no. } \\
\text { of patients }\end{array}$ & $\begin{array}{c}\text { True } \\
\text { positive, no. } \\
\text { of patients }\end{array}$ & $\begin{array}{c}\text { True } \\
\text { negative, no. } \\
\text { of patients }\end{array}$ & $\begin{array}{c}\text { False } \\
\text { positive, no. } \\
\text { of patients }\end{array}$ & $\begin{array}{c}\text { False } \\
\text { negative, no. } \\
\text { of patients }\end{array}$ & $\begin{array}{c}\text { Sensitivity (\%) } \\
(95 \% \mathrm{CI})\end{array}$ & $\begin{array}{c}\text { Specificity (\%) } \\
(95 \% \mathrm{Cl})\end{array}$ & $\begin{array}{l}\text { PPV } \\
(\%)\end{array}$ & $\begin{array}{l}\text { NPV } \\
(\%)\end{array}$ \\
\hline Angulo P [14] & 23 & 1 & 22 & 0 & 0 & $\begin{array}{c}100.0 \\
(95.6-100.0)\end{array}$ & $\begin{array}{c}100.0 \\
(95.6-100.0)\end{array}$ & 100.0 & 100.0 \\
\hline Baskan 0 [15] & 39 & 1 & 37 & 0 & 1 & $\begin{array}{c}50.0 \\
(47.4-52.6)\end{array}$ & $\begin{array}{c}100.0 \\
(97.4-100.0)\end{array}$ & 100.0 & 97.4 \\
\hline Berstad AE [16] & 39 & 1 & 38 & 1 & 0 & $\begin{array}{c}100.0 \\
(97.4-100.0)\end{array}$ & $\begin{array}{c}97.4 \\
(91.2-100.0)\end{array}$ & 50.00 & 100.0 \\
\hline Muir AJ [17] & 234 & 3 & 231 & 0 & 0 & $\begin{array}{c}100.0 \\
(99.3-100.0)\end{array}$ & $\begin{array}{c}100.0 \\
(99.3-100.0)\end{array}$ & 100.0 & 100.0 \\
\hline Samuel MT [18] & 20 & 10 & 10 & 0 & 0 & $\begin{array}{c}100.0 \\
(84.2-100.0)\end{array}$ & $\begin{array}{c}100.0 \\
(84.2-100.0)\end{array}$ & 100.0 & 100.0 \\
\hline Tenca A [19] & 59 & 4 & 55 & 0 & 0 & $\begin{array}{c}100.0 \\
(96.6-100.0)\end{array}$ & $\begin{array}{c}100.0 \\
(96.6-100.0)\end{array}$ & 100.0 & 100.0 \\
\hline Tenca A [20] & 48 & 7 & 41 & 0 & 0 & $\begin{array}{c}100.0 \\
(94.4-100.0)\end{array}$ & $\begin{array}{c}100.0 \\
(94.5-100.0)\end{array}$ & 100.0 & 100.0 \\
\hline Weber C [21] & 69 & 6 & 67 & 0 & 2 & $\begin{array}{c}75.0 \\
(71.4-78.5) \\
\end{array}$ & $\begin{array}{c}100.0 \\
(96.5-78.6)\end{array}$ & 100.0 & 97.1 \\
\hline Total & 531 & 33 & 501 & 1 & 3 & $\begin{array}{c}98.9 \\
(98.6-99.3) a\end{array}$ & $\begin{array}{c}99.9 \\
(99.6-100) a\end{array}$ & $97.1 \mathrm{~b}$ & $99.4 b$ \\
\hline
\end{tabular}

MRCP - magnetic resonance cholangiopancreatography, NPV - negative predictive value, PPV - positive predictive value, PSC - primary sclerosing cholangitis

aPooled from random effects model meta-analysis,

${ }^{b}$ Crude PPV and NPV calculation

ty was 98.9\% (95\% CI: 98.6-99.3\%) (Table 2, Fig. 2). Anatomic details of cholangiocarcinoma cases (i.e., intrahepatic, hilar, extrahepatic) were available in only 4 studies. Hilar cholangiocarcinoma cases missed by MRI/MRCP were diagnosed as beading irregularities of the central hepatic ducts, and extrahepatic cholangiocarcinoma cases were diagnosed as "PSC-related diffuse stricture of the extrahepatic bile ducts". All 9 cases with available details were diagnosed later with endoscopic retrograde cholangiopancreatography (ERCP)-guided brush cytology. Pooled specificity was close to $100 \%$. Only 1 false-positive case was found in the literature with limited details on secondary testing or follow-up (Table 2) [16]. 


\begin{tabular}{|c|c|c|c|}
\hline Author & & $\mathrm{ES}(95 \% \mathrm{Cl})$ & Weight \\
\hline Angulo $P$ & $\rightarrow$ & $1.00(0.96,1.00)$ & 2.39 \\
\hline Baskan 0 & * & $0.50(0.47,0.53)$ & 1.72 \\
\hline Berstad AE & $\rightarrow$ & $1.00(0.97,1.00)$ & 6.88 \\
\hline Muir AJ & - & $1.00(0.99,1.00)$ & 82.51 \\
\hline Samuel MT & $\longrightarrow$ & $1.00(0.84,1.00)$ & 0.18 \\
\hline Tenca A & 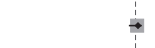 & $1.00(0.97,1.00)$ & 3.93 \\
\hline Tenca A & $\rightarrow$ & $1.00(0.94,1.00)$ & 1.49 \\
\hline Weber C & + & $0.75(0.71,0.79)$ & 0.90 \\
\hline Overall $(R=99.6 \%, p=0.000)$ & 1 & $0.99(0.99,0.99)$ & 100.00 \\
\hline
\end{tabular}

ES - effect size, MRCP - magnetic resonance cholangiopancreatography, MRI - magnetic resonance imaging, PSC - primary sclerosing cholangitis

Fig. 2. Forest plot of MRI/MRCP sensitivity and specificity to identify cholangiocarcinoma in PSC

\section{Meta-regression}

Meta-regression revealed that neither year of publication, study design, nor sample size had a significant effect on the observed sensitivity estimates $(P=0.9$, 0.3 , and 0.3 , respectively).

\section{Assessment of bias and heterogeneity}

Risk of bias measured with the Newcastle-Ottawa Scale score ranged from 4 to 6 points (Table 3). Most studies lost points due to limited representativeness of the general PSC population, incomplete details in the methods, or failure to describe salient patient characteristics. The $I^{2}$ for the model was $99.6 \%$.

The funnel plot showed a paucity of studies with low sensitivity and small sample size. No studies had prospective surveillance (e.g., follow-up encounter and imaging in 1 year), potentially reducing the frequency of early PSC disease with false-negative results (Fig. 3).

\section{Discussion}

Our study shows that MRI/MRCP has specificity close to $100 \%$, with only one false-positive case reported in the literature. Sensitivity was also high at around $98.9 \%$, with only 3 false-negative results. Even though our findings are significantly limited by primary studies' characteristics (i.e., retrospective design, selection bias, and lack of follow-up), they suggest that screening PSC patients with MRI/MRCP is an attractive screening strategy that merits rigorous evaluation in prospective studies. Periodic MRI/MRCP has the potential to decrease the high cancer-related mortality associated with cholangiocarcinoma.

Two meta-analyses have previously reviewed the diagnostic yield of MRI/MRCP in biliary strictures.
Table 3. Newcastle-Ottawa Assessment of Bias

\begin{tabular}{lcccc}
\hline First author & Selection & $\begin{array}{c}\text { Compa- } \\
\text { rability }\end{array}$ & $\begin{array}{c}\text { Exposure } \\
\text { or outcome }\end{array}$ & $\begin{array}{c}\text { Total } \\
\text { (max 9 pts) }\end{array}$ \\
\hline Angulo P [14] & 2 & 1 & 3 & 6 \\
\hline Baskan 0 [15] & - & - & - & N/A \\
\hline Berstad AE [16] & 2 & 0 & 2 & 4 \\
\hline Muir AJ [17] & 2 & 0 & 3 & 5 \\
\hline Samuel MT [18] & 2 & 1 & 3 & 6 \\
\hline Tenca A [19] & 2 & 0 & 2 & 4 \\
\hline Tenca A [20] & 3 & 0 & 3 & 6 \\
\hline Weber C [21] & 1 & 1 & 2 & 4 \\
\hline
\end{tabular}

Max - maximum, pts - points

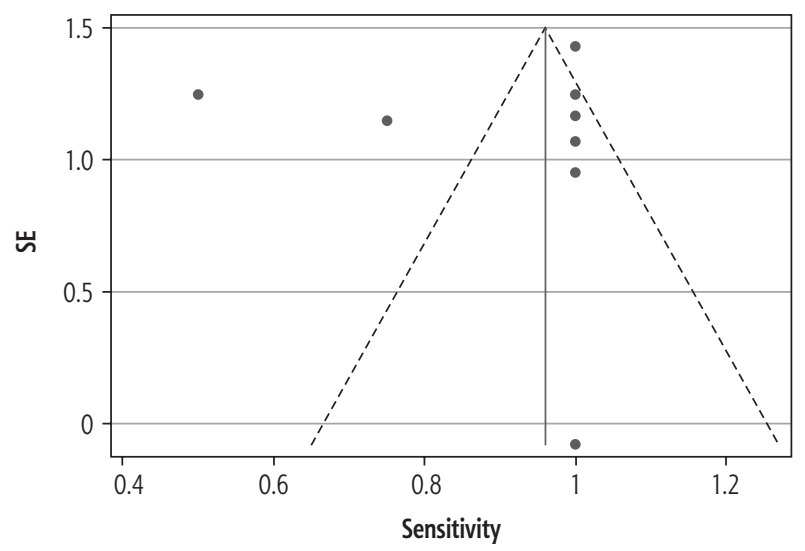

SE - standard error

Fig. 3. Funnel plot for included studies with pseudo $95 \% \mathrm{Cl}$

Dave et al. [12] reviewed the value of MRI/MRCP to identify PSC in patients with biliary obstruction and cholestasis, demonstrating an extremely high specificity (0.94 [95\% CI: 0.86-0.98]) and a positive likelihood ratio (15.5 [95\% CI: 6.2-38.1]). Romagnuolo et al. [13] concluded that MRI/MRCP is a noninvasive imaging 
test with excellent overall sensitivity and specificity to demonstrate the presence and anatomy of any biliary obstruction. However, it seems less sensitive for differentiating malignant from benign obstruction [13]. Given its noninvasive nature, MRI/MRCP can be extremely useful in the long-term care of patients [20].

Given the low prevalence of PSC in the general population, performing a randomized clinical trial with enough power to show mortality reduction with PSC screening would be extremely difficult. Our metaanalysis shows that use of high-quality imaging (MRI/ MRCP) followed by endoscopic confirmation can identify cholangiocarcinoma with high specificity (close to $100.0 \%$ ) and sensitivity (98.9\%).

The high specificity of MRI/MRCP in the diagnosis of cholangiocarcinoma has to be balanced with the number of false-negative cases missed. Missing early cholangiocarcinoma will substantially limit the therapeutic options. After a false-negative test, patients might return to their physician months later beyond the window for surgical treatment (i.e., resection or liver transplant). Due to the wide range of clinical presentations, even in advanced stages, routine screening prior to the symptomatic phase may allow early diagnosis of cholangiocarcinoma in patients with known risk factors [24]. Diffusion-weighted imaging enhances the diagnostic sensitivity of MRI/MRCP for detection of tumors within dilated or obstructed ducts [25]. If a patient has a confirmed cholangiocarcinoma lesion, MRI/MRCP can help determine resectability. Our review focused on MRI/MRCP as the primary imaging. Our estimates are likely inflated by the fact that many programs required complementary testing (i.e., CA 19-9, ERCP) when warranted by initial findings. Limited reporting of the use of brushings, biopsies, or other endoscopic and percutaneous testing prevented any stratified analysis (no details were reported on conference abstracts). This approach can improve the sensitivity of screening programs based solely on MRI/ MRCP [26]. In real practice, once a high-risk lesion is identified with cross-sectional imaging, the chances of confirming cholangiocarcinoma with histology are still suboptimal. In a meta-analysis published in 2016, the pooled sensitivity of single-operator cholangioscopy for diagnosis of cholangiocarcinoma in patients with PSC was only $65 \%$ (95\% CI: 35-87\%) [26]. These data are particularly relevant in health care facilities with limited access to advanced endoscopy, such as cholangioscopy with targeted biopsies and fluorescence in situ hybridization analysis. MRI/MRCP can therefore provide enough evidence for referral to a facility with ERCP-cholangioscopy capabilities.
In addition to imaging performance, individual patient characteristics and local practice patterns play a critical role in diagnosing cholangiocarcinoma. Clinical characteristics such as weight loss, smoking, and initial presentation of PSC are all clinical variables that should increase suspicion from clinicians ordering imaging studies.

\section{Strengths and limitations}

To our knowledge, this is the first published review on diagnostic performance of MRI/MRCP in PSC to include a meta-analysis for cholangiocarcinoma cases and to estimate pooled sensitivity and specificity rates. Our results were complemented with meta-regression analysis, comparing baseline modifiers from study design. The inclusion criteria were permissive for all PSC, but excluded patients presenting with biliary obstruction from other conditions and small studies with fewer than 20 patients. The database review was comprehensive, including meeting abstracts and references. The funnel plot suggested low chances of publication bias (only a paucity of small sample-size studies).

Our study is limited by the poor quality of primary studies. Most studies were designed to measure the accuracy of MRI/MRCP to diagnose PSC, not to diagnose cholangiocarcinoma. This is a potential source of selection bias. The majority of studies were retrospective (7/8) and none had a follow-up encounter to confirm false-negative tests. Study quality rated by the Newcastle-Ottawa Scale is considered moderate to low.

Details on cirrhosis of patients with PSC were limited and prevented us from performing stratified analysis. These details would have been very informative as patients with cirrhosis, according to guidelines, should already be receiving imaging screening every 6 months to identify hepatocellular carcinoma. Patients without cirrhosis are the ones who can benefit from additional imaging to identify early cholangiocarcinoma.

Finally, the authors recognize the potential for underreporting and underestimating studies with false-negative results. Lack of follow-up imaging results may have missed patients who developed cholangiocarcinoma after MRI/MRCP was performed during the study period.

Our $I^{2}$ value suggests very low chances of heterogeneity not explained by chance.

\section{Conclusions}

This meta-analysis shows that MRI/MRCP is a very sensitive and specific tool to identify cholangiocarcino- 
ma cases in patients with PSC. Further studies should measure MRI/MRCP sensitivity in a prospective fashion, assess the efficacy of MRI as a screening tool in patients with PSC and cirrhosis, and establish the best strategies to combine MRI/MRCP with ERCP-guided brushings and biopsies. These uncertainties should be answered before screening programs are implemented while parallel studies explore novel biomarkers, assess chemoprevention, and refine current imaging protocols.

\section{Disclosure}

The authors report no conflict of interest.

\section{References}

1. Soetikno RM, Lin OS, Heidenreich PA, et al. Increased risk of colorectal neoplasia in patients with primary sclerosing cholangitis and ulcerative colitis: a meta-analysis. Gastrointest Endosc 2002; 56: 48-54.

2. Lazaridis KN, LaRusso NF. Primary sclerosing cholangitis. N Engl J Med 2016; 375: 1161-1170.

3. Jepsen P, Vilstrup H, Tarone RE, et al. Incidence rates of intraand extrahepatic cholangiocarcinomas in Denmark from 1978 through 2002. J Natl Cancer Inst 2007; 99: 895-897.

4. Antwi SO, Mousa OY, Patel T. Racial, ethnic, and age disparities in incidence and survival of intrahepatic cholangiocarcinoma in the United States; 1995-2014. Ann Hepatol 2018; 17: 604-614.

5. Lazaridis KN, Gores GJ. Primary sclerosing cholangitis and cholangiocarcinoma. Semin Liver Dis 2006; 26: 42-51.

6. Chapman R, Fevery J, Kalloo A, et al. Diagnosis and management of primary sclerosing cholangitis. Hepatology 2010; 51: 660-678.

7. Khaderi SA, Sussman NL. Screening for malignancy in primary sclerosing cholangitis (PSC). Curr Gastroenterol Rep 2015; $17: 17$.

8. Patel AH, Harnois DM, Klee GG, et al. The utility of CA 19-9 in the diagnoses of cholangiocarcinoma in patients without primary sclerosing cholangitis. Am J Gastroenterol 2000; 95: 204-207.

9. Fulcher AS, Turner MA, Franklin KJ, et al. Primary sclerosing cholangitis: evaluation with MR cholangiography-a case-control study. Radiology 2000; 215: 71-80.

10. Charatcharoenwitthaya P, Enders FB, Halling KC, et al. Utility of serum tumor markers, imaging, and biliary cytology for detecting cholangiocarcinoma in primary sclerosing cholangitis. Hepatology 2008; 48: 1106-1117.

11. Moher D, Liberati A, Tetzlaff J, et al. Preferred reporting items for systematic reviews and meta-analyses: the PRISMA statement. BMJ 2009; 339: b2535.

12. Dave M, Elmunzer BJ, Dwamena BA, et al. Primary sclerosing cholangitis: meta-analysis of diagnostic performance of MR cholangiopancreatography. Radiology 2010; 256: 387-396.

13. Romagnuolo J, Bardou M, Rahme E, et al. Magnetic resonance cholangiopancreatography: a meta-analysis of test performance in suspected biliary disease. Ann Intern Med 2003; 139: 547557.

14. Angulo P, Pearce DH, Johnson CD, et al. Magnetic resonance cholangiography in patients with biliary disease: its role in primary sclerosing cholangitis. J Hepatol 2000; 33: 520-527.
15. Baskan O, Erol C, Sahingoz Y. Portal biliopathy, magnetic resonance imaging and magnetic resonance cholangiopancreatography findings: a case series. Gastroenterol Rep (Oxf) 2016; 4: 68-72.

16. Berstad AE, Aabakken L, Smith HJ, et al. Diagnostic accuracy of magnetic resonance and endoscopic retrograde cholangiography in primary sclerosing cholangitis. Clin Gastroenterol Hepatol 2006; 4: 514-520.

17. Muir AJ, Taghipour M, Hassanzadeh E, et al. A risk prediction score based on magnetic resonance cholangiopancreatography (MRCP) accurately predicts disease progression in patients with primary sclerosing cholangitis (PSC). [Abstract]. Hepatology 2017; 66: 81A.

18. Samuel MT, Fowler K, Lin M, et al. Clinical and radiologic predictors of progression of primary sclerosing cholangitis (PSC): A pilot study [Abstract]. Gastroenterology 2017; 152: S1061.

19. Tenca A, Lind K, Lantto E, et al. Comparison of magnetic resonance cholangiopancreatography and endoscopic retrograde cholangiography in disease severity assessment of primary sclerosing cholangitis: Impact on prognosis. [Abstract]. United European Gastroenterol J 2016; 4: A407-A408.

20. Tenca A, Mustonen H, Lind $\mathrm{K}$, et al. The role of magnetic resonance imaging and endoscopic retrograde cholangiography in the evaluation of disease activity and severity in primary sclerosing cholangitis. Liver Int 2018; 38: 2329-2339.

21. Weber C, Kuhlencordt R, Grotelueschen R, et al. Magnetic resonance cholangiopancreatography in the diagnosis of primary sclerosing cholangitis. Endoscopy 2008; 40: 739-745.

22. Stang A. Critical evaluation of the Newcastle-Ottawa scale for the assessment of the quality of nonrandomized studies in meta-analyses. Eur J Epidemiol 2010; 25: 603-605.

23. Higgins JP, Thompson SG, Deeks JJ, et al. Measuring inconsistency in meta-analyses. BMJ 2003; 327: 557-560.

24. Hennedige TP, Neo WT, Venkatesh SK. Imaging of malignancies of the biliary tract - an update. Cancer Imaging 2014; 14 : 14.

25. Jhaveri KS, Hosseini-Nik H. MRI of cholangiocarcinoma. J Magn Reson Imaging 2015; 42: 1165-1179.

26. Njei B, McCarty TR, Varadarajulu S, et al. Systematic review with meta-analysis: endoscopic retrograde cholangiopancreatography-based modalities for the diagnosis of cholangiocarcinoma in primary sclerosing cholangitis. Aliment Pharmacol Ther 2016; 44: 1139-1151. 